\title{
Interscalar Design and Health Research Partnership: Research Integration Into Curriculum and Practice
}

\section{SHANE IDA SMITH}

University of Arizona,

School of Architecture

ESTHER M. STERNBERG

University of Arizona,

College of Medicine

\section{ARTHUR CHRIS NELSON}

University of Arizona,

School of Planning

\section{MARY C. HARDIN}

University of Arizona,

School of Architecture
Numerous built environment factors have a negative effect upon human health and wellbeing, including lack of natural daylighting, light trespass, poor air quality, poor water quality, damaging noise pollution, uncontrolled thermal conditions, constraints and limitations on physical mobility, disorienting surroundings, amongst others. A new vision for environmental health extends beyond the traditional removal of negative factors that cause illness and disease to embrace aspects of the built and natural environment that support physical health and emotional wellbeing.

Our interdisciplinary team of biomedical, public health, integrative medicine, architecture, landscape architecture, and planning disciplines is conducting research at various scales to inform innovative concepts for addressing the interrelated physiological and biopsychosocial challenges. Novel correlations between health and wellbeing with built environment phenomena are particularly applicable for multiple aspects of design, including environmental quality, integrated natural systems, sensory environments, and safety. Integration of social connectedness and physical activity in design and urban planning are also implicated in this research.

The research framework presented here focuses on four significant aspects of this ongoing multidisciplinary effort: individual scale, community scale, building scale, and integrated outcomes for implementation of education and curriculum into practice. Primary physiological impacts of built environment factors upon health and wellbeing are documented at the individual level while biopsychosocial impacts are investigated at the community level with a focus on resiliency. These research results are implemented into academic design/build outreach practice for underserved communities, and also inform core fundamentals of environmental factors for dissemination in architecture curriculum technical courses.

Scientific correlations of biopsychosocial metrics to the built and natural environmental factors are made by quantifying pre- and post-occupancy studies to assess the impact of sustainable design on human physical and emotional health and wellbeing. An example of this approach is in our previous study showing that sustainable design positively impacted 
two different measures of physiological stress response. ${ }^{1}$ The research is conducted by the use of non-invasive, unobtrusive mobile health devices and heart rate variability monitors, which allow for specific environmental attributes such as light (intensity, wavelength, glare, circadian rhythm), noise, temperature, airflow, etc. to be measured and correlated with physiological responses (stress and relaxation response) and psychological momentary experience sampling. The relationships between these health responses to environmental conditions are analyzed using Big Data Analytics to obtain time and locational attributes of both health and environmental data. The quantitative aspects of the research are cross-correlated with qualitative documentation of the designed environment affecting conditional responses in human health. Through parallel interscalar research investigations, the projected impacts of new knowledge surrounding health and wellbeing within the built environment will expand current paradigms for integrated design processes and methods. The innovative core of this research is the science of brain-immune-environmental interactions. The results of this research intend for the evidence needed to support holistic health and wellbeing in the design, construction, and maintenance of built environments. The critical position that these research activities assert is a prerogative for deep partnerships between biomedical and health professionals alongside those who design and steward the built environment.

\section{INTRODUCTION}

Basic principles of architectural design predominantly focus on ocularcentric aspects of human experience. Such cultural codes where sight prevails at the forefront of design thinking tend to suppress qualities of space and place for alternate sentient human experience. This facet of design theory is explored in the work of architect Juhani Pallasmaa through a phenomenological positioning. Pallasmaa situates the experience of architecture through more holistic concepts of sensation, including haptic and aural, in addition to the visual conditions of the environment. ${ }^{2}$ Shifts in architectural theory towards encompassing phenomenology exemplify a contemporary mode by which human experience influences design practice and pedagogical methods engaged in design curricula.

The introduction of physiological and biopsychosocial human health measures in built environment research expands the potentials for deriving useful collective information for societal wellbeing. Our interscalar approach to research on built environment human health and wellbeing integrates both the individual and structuralist accounts, and will make use of the outcomes through codification of design principles for education and practice.

\section{INDIVIDUAL SCALE}

The World Health Organization's definition of health in the 21st Century states that health is more than the absence of disease. ${ }^{3}$ It encompasses going beyond the traditional removal of negative factors that cause illness to embrace aspects of the built and natural environment that support physical health and emotional wellbeing.

A fundamental understanding of human responses to both positive and negative environmental factors is therefore important for design professionals to instantiate these principles into practice and to design environments for wellbeing at all scales.

It is well known that many aspects of the physical environment can either stress or calm. Broadly speaking, this includes all aspects of perception through each of the five senses vision, hearing, smell, touch, and even taste. Activities also impact the stress and relaxation responses. Thus what one sees, hears, smells, touches and does in a space can all influence the brain and body's stress and relaxation responses. ${ }^{4}$

It is important to note that the brain's stress response is essential to life, and is the organism's main strategy to get out of danger, focus attention, and perform at peak. The goal therefore is not to get rid of stress, but to optimize the stress response to match the 
activity at hand. There is an "inverted U shaped curve" - and upside-down $U$ that relates activity of the stress response to performance. At the far left of the curve one is totally relaxed and the stress response is tuned down very low - one is half asleep and not performing at peak. In order to perform at peak, the stress response needs to be turned on optimally. Performance fails when the stress response goes into overdrive, or lasts too long once the danger has passed.

Another important foundational principle is the role of the brain's stress response on the immune response: the science of the mind-body connection. ${ }^{5}$ A wealth of research has established the many ways in which the brain and immune systems communicate. It is well known that chronic stress can prolong wound healing, increase susceptibility and severity of viral infections, speed chromosomal aging and speed cancer growth by impairing the immune system's ability to fight disease. This occurs through excess release of the antiinflammatory stress hormone cortisol. In contrast, integrative medicine mind-body interventions, such as yoga, meditation, tai chi, exercise can all reverse the negative effects of stress on the body, optimize emotional and physical health and prevent disease. Social support is also important in health. On an individual basis, holding hands with a loved one significantly lowers the stress response and studies have shown that persons with a greater number of positive social interactions are healthier ${ }^{6}$, while isolated individuals, especially the elderly are more prone to disease. ${ }^{7}$

Nature, or biophilia, is also an important stress reducer, where increasing numbers of plants in the environment and views of nature have been shown to significantly reduce stress and enhance the effects of activities, such as exercise. ${ }^{8}$ Design professionals can help individuals fine-tune their stress response passively, simply by designing the built environment at all scales to stimulate or relax the senses, and to help foster healthy activities, such as exercise, meditation, and social support. This is familiar to all who have been to a spa, versus an airport or typical hospital.

Attributes of the built environment can have negative or positive effects on human health and wellbeing [Fig. 1]. Negative impacts include: lack of natural day-lighting, glare; noise; foul odors; uncontrolled thermal conditions; poor air quality; poor water quality; constraints and limitations on physical mobility, disorienting surroundings, amongst others. Positive

Figure 1: Interscalar Design and Health Research Integration Into Curriculum and Practice.

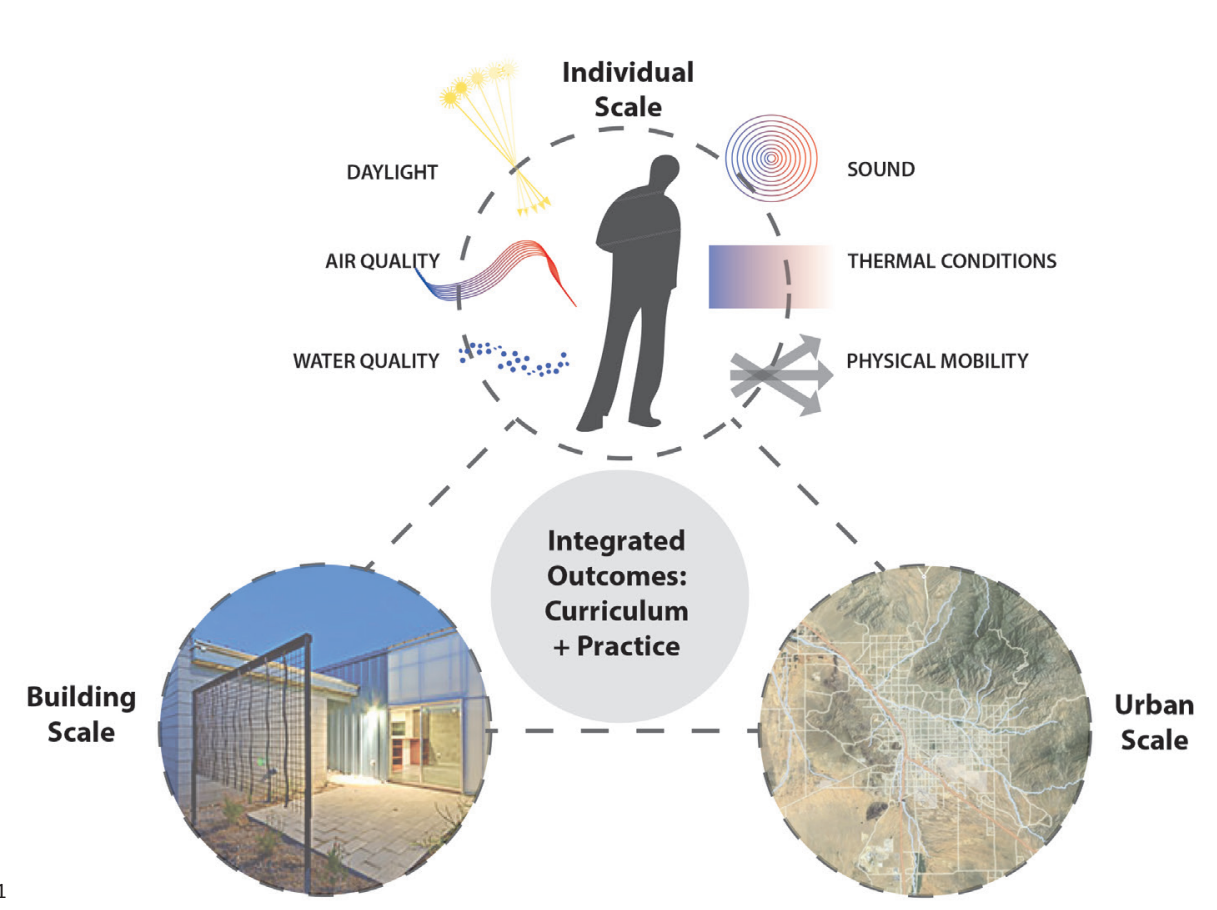


impacts include optimal levels and qualities of light; optimal sound level, soothing music, nature sounds; controlled thermal conditions; way-finding; and areas for social support.

Our interdisciplinary team's research framework focuses on development of methods and technologies to sensitively measure the impact of many environmental attributes on human psychological, behavioral, physiological, and even molecular responses. These can be applied to quantify the impact of the built and natural environment on human responses at all scales: individual, building, and community scales.

Scientific correlations of biomedical metrics to the built and natural environmental factors are made by quantifying pre- and post-occupancy studies to assess the impact of sustainable design on human physical and emotional health and wellbeing. The research is conducted by the use of non-invasive, unobtrusive mobile health devices such as heart rate variability monitors, which measure the balance between the two components of the nervous system that detect the stress and relaxation responses - the sympathetic (stress) and parasympathetic (relaxation) responses. The latest generation of such monitors also detect activity, posture, sleep quality and even altitude at such sensitive levels that one can detect whether a person is climbing stairs. Other devices can be used to measure psychological responses, so-called momentary experience sampling. Data from such mobile devices can be used to track the human experience in real-time and real-place. Eventually new technologies will allow such data streams to be linked to specific environmental attributes that the person experiences, such as light (intensity, wavelength, glare, circadian rhythm), noise, temperature, airflow, etc. The relationships between these health responses to environmental conditions are analyzed using Big Data Analytics to obtain time and locational attributes of both health and environmental data. The quantitative aspects of the research are cross-correlated with qualitative documentation of the designed environment affecting conditional responses in human health.

The goal of developing such quantitative methods is to inform design principles to optimize the built environment for health and wellbeing. We are at an exciting juncture in the fields of design and mobile, personalized health, where the data gleaned from such tools will help design professionals optimize their designs for health and wellbeing, instantiating the World Health Organization's goals of viewing health as far more than the absence of disease, and encompassing the impact of all aspects for a person's environment in their health.

\section{COMMUNITY SCALE}

Planning engages multiple scales of activity external to physical structures themselves. Research at the University of Arizona will reshape the curriculum and ultimately practice as it relates to the nature of urban form from the neighborhood to the regional scales. It begins with modeling dimensions of urban form. It continues with identifying opportunities to reshape the built environment at all scales through interventions in the life cycle of structures and built landscapes.

The point of departure for urban form research is recent work by Reid Ewing and Shima Hamidi (2014) $)^{9}$ published by the National Cancer Institute: Measuring Urban Sprawl and Validating Sprawl Measures. ${ }^{10}$ They modeled four dimensions of urban form to create an overall index of urban sprawl-which is recast as the Ewing/Hamidi Urban Form Index below. Those dimensions and their component parts are:

Density

1. Gross density of urban and suburban census tracts;

2. Percentage of the population living at low suburban densities (less than 1,500 persons per square mile);

3. Percentage of the population living at medium to high urban densities (between 1,500 and 12,500 persons per square mile); 
4. Urban density based on the National Land Cover Database; and

5. Gross employment density of urban and suburban census tracts.

Mixed-Use

1. Countywide average job-population balance (jobs/population);

2. Countywide degree of job mixing (based on an entropy model); and

3. Countywide average Walk Score. ${ }^{11}$

Centering/Agglomeration of Activities

1. The coefficient of variation in census block group population densities, defined as the standard deviation of block group densities divided by the average density of block groups (where the more variation in densities around the mean, the more centering and/or subcentering exists within the county); and

2. The coefficient of variation in census block group employment densities, defined as the standard deviation of block group densities divided by the average density of block groups (where the more variation in densities around the mean, the more centering and/or subcentering exists within the county);

3. Percentage of county population in CBD or sub-centers; and

4. Percentage of county employment in CBD or sub-centers.

Street Accessibility

1. Intersection density for urban and suburban census tracts within the county, excluding rural tracts with gross densities of less than 100 persons per square mile; and

2. Percentage of 4-or-more-way intersections, again excluding rural tracts.

Ewing and Hamidi summed the individual scores which has the effect of giving each component within each respective dimension equal weight, then giving each dimension equal weight ( 25 percent each) in calculating an overall raw score. ${ }^{12}$ They then transformed the overall score into an index having a mean of 100 and a standard deviation of 25 (similar to the Stanford-Binet intelligence quotient score). More compact landscapes have index scores above 100 (see the left panel in Figure 2-Arlington County, Virginia in this case) and more sprawling ones have scores below 100 (see the right panel in Figure 2-Oglethorpe County, Georgia, for example).

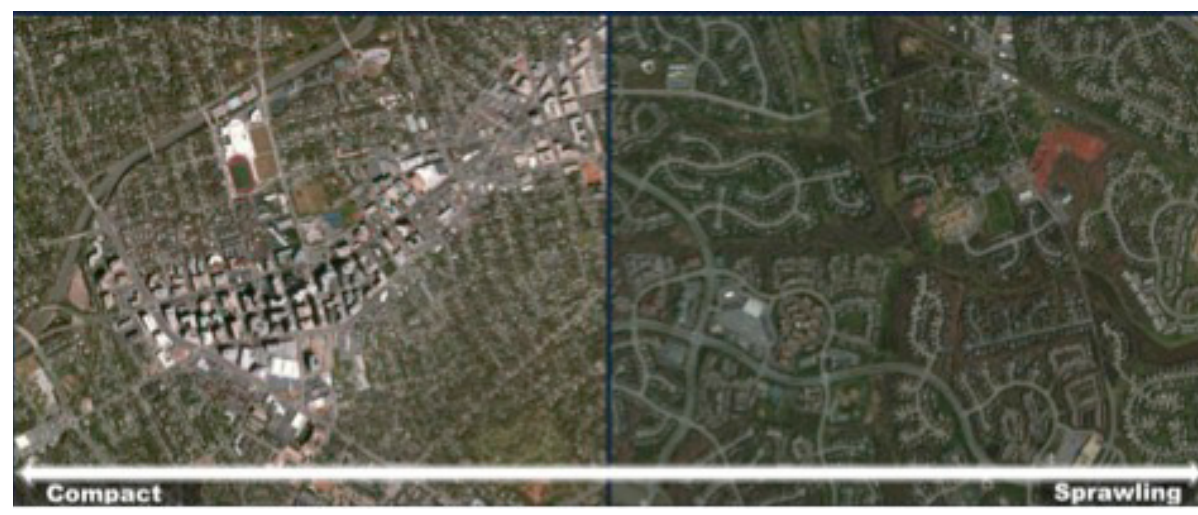

Figure 2: Continuum of Compact and Sparwling Urban Form Conceptualized. (Image: Ewing and Hamidi, 2014)
We have tested the Ewing/Hamidi Urban Form Index ${ }^{13}$ in a variety of applications and find it rather robust. For instance, in using it as a predictor (among many control variables) for change in metropolitan- and county-level job change between 2000 and 2010 (meeting 
all relevant statistical tests with a large coefficient of determination), elasticity outcomes indicated that a 10-percent increase in the Urban Form Index increased jobs by 0.6-percent, which is nearly the same outcome measured in different ways by two independent teams of econometricians. A team of University of Arizona researchers is being assembled to propose research that applies the Urban Form Index to a variety of public health concerns at metropolitan, county, and neighborhood scales.

This research will help advance the use of scenario planning whereby outcomes to alternative urban forms resulting in higher Urban Form Index scores can be estimated to help guide long-range planning to achieve preferred public health and other outcomes through explicit changes to urban form.

We have pioneered methods to estimate when different types of the built environment may become opportunities for redevelopment from the level of the parcel to an entire metropolitan area. ${ }^{14}$ For instance, over the next 30 years more than half of all nonresidential structures and up to a fifth of all residential structures in growing metropolitan areas will be torn down and replaced, repurposed through substantial rehabilitation, or in other ways recycled. Using a parcel-based analysis allowing for assembly of parcels to different scales, planners can identify when large segments of commercial corridors, neighborhoods, nodes or other landscapes may become opportunities for redevelopment at about the same time. We have also developed models to help understand the timing of market-driven redevelopment where markets are currently unable to justify such redevelopment. These tools calibrate the nature of public-sector intervention needed to accelerate redevelopment so that the built landscape may be reshaped earlier than later to achieve broad public policy objectives during a planning horizon. ${ }^{15}$

The next logical step is to merge the Urban Form Index with models to facilitate the interscalar reshaping of the built environment so that redevelopment decisions are made with improved appreciation of benefits. The merging would occur after research demonstrates the utility of increasing the Urban Form Index score substantially through the redevelopment process in ways that improve public health, economic, quality-of-life, and related outcomes. Once the calibration is done, the Urban Form Index can be merged into scenario planning packages such as Envision Tomorrow Plus. ${ }^{16}$ The merged models can then be included in planning and design-based curriculums to better inform the next generation of practitioners on the benefits of alternative designs of the built environment at widely varying scales.

\section{BUILDING SCALE}

The University of Arizona School of Architecture engages students with design-build projects through the Drachman Design Build Coalition (DDBC), an entity that identifies local sites in Tucson for single-family affordable housing development to execute design, construction, and post-occupancy activities. This design-build outreach component of the professional architecture curriculum allows for both the integration of research in application as well as the translation of classroom learning into practice.

While most DDBC structures are developed with innovative building technologies, including alternative construction materials and methods for passive environmental performance, more recent DDBC houses will integrate outcomes from the human health and wellbeing research to inform unique design strategies [Fig. 3]. Fundamental attributes for human health in built environment design, including natural daylighting, sound control, thermal conditions, as well as air and water quality measures, are established as guiding principles for future design development and post-occupancy analysis studies of DDBC houses. Prior DDBC projects formalized research outcomes for energy and water conservation strategies in affordable housing design, which will continue to be valid and progressed for further development towards net-zero energy and net-zero water dwellings. However, 

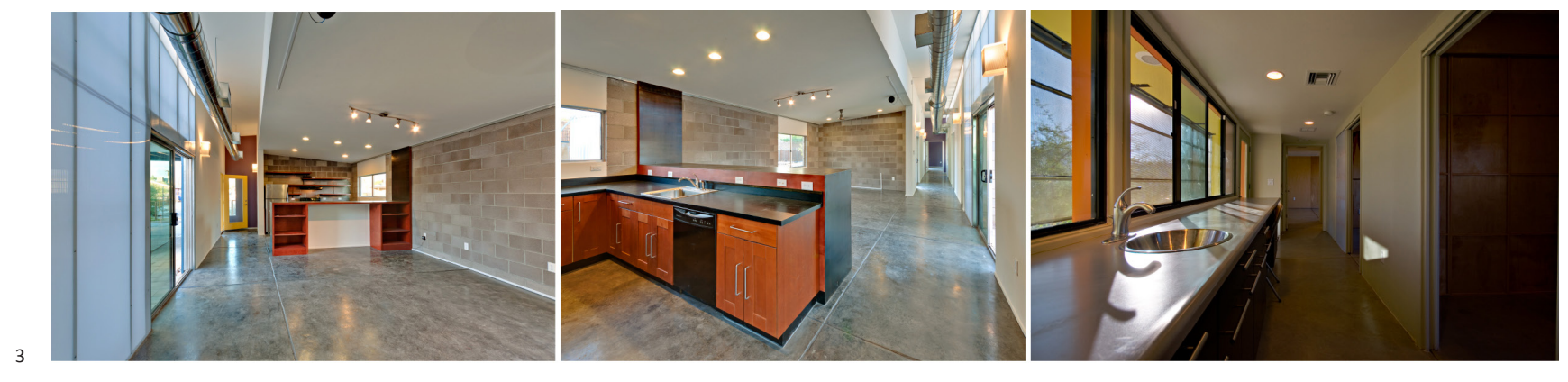

additional focus for research in DDBC projects will equally emphasize modes for measuring effectiveness of the designs for health benefit.

The DDBC projects also negotiate the community scale public health research by means of assessing potentials for infill sites and determining optimal setbacks, building form, and programmatic relationships as contextual response. The site availability for affordable housing projects can be burdened with particular environmental challenges that enhance the necessity for addressing human health and wellbeing in such conditions through the design and construction techniques. For instance, some of the sites available for these projects may be located adjacent or near Industrial zoning, where noise and air pollution may prevail. Other sites for affordable housing projects may be zero-lot line infill conditions with limited access to adequate daylight.

In addition, particular tools and methods are currently being developed to measure and analyze building-scale design concepts against integration of health and wellbeing attributes prevalent in such environments. These methods include the analysis of design attributes for interior habitable spaces of digital models and built spaces with fisheye lens photographs and evaluative tools [Fig. 4].

By establishing 360-degree view-factor images of a human individual's interface with the interior environment, metrics can be calculated for the quantification of particular environmental attributes that can significantly impact human health. The view-factor calculations of these attributes may include natural daylight exposure, exterior views, bright materials, dark materials, plants, etc., and are intended to provide baseline relevance of spatialized influence upon human experience. The integration of design factors specific for human health at the building scale is thus accurately documented for correlation with the individual metrics of human stress response and localized environmental sensing. This methodological framework stitches together building-scale factors as causal measures influencing environmental data (such as photometric, air quality, noise vibration, and thermal conditions) against individual-scale measures based upon heart-rate variability and stress response. This framework inserts both the human's response to spatial and material design qualities as well as environmental conditions to inform building scale principles.

\section{INTEGRATED OUTCOMES FOR CURRICULUM AND PRACTICE}

Cross-linking the individual, building, and community scales of human health and wellbeing research in this interdisciplinary effort is informing a series of emerging concepts applicable for design fundamentals. The significance of the individual scale research informs the biophysiological responsiveness to environmental design attributes for optimal human wellbeing that encompasses physical, emotional, and mental aspects functioning from a phenomenological positioning. The significance of the community and urban scale research informs both physical mobility and social connectedness design attributes for broader public health, functioning from a structuralist positioning. The significance of the building scale research offers a negotiation between the spectrum of individual and collective human experience and physiological response. The building scale addresses the health of 

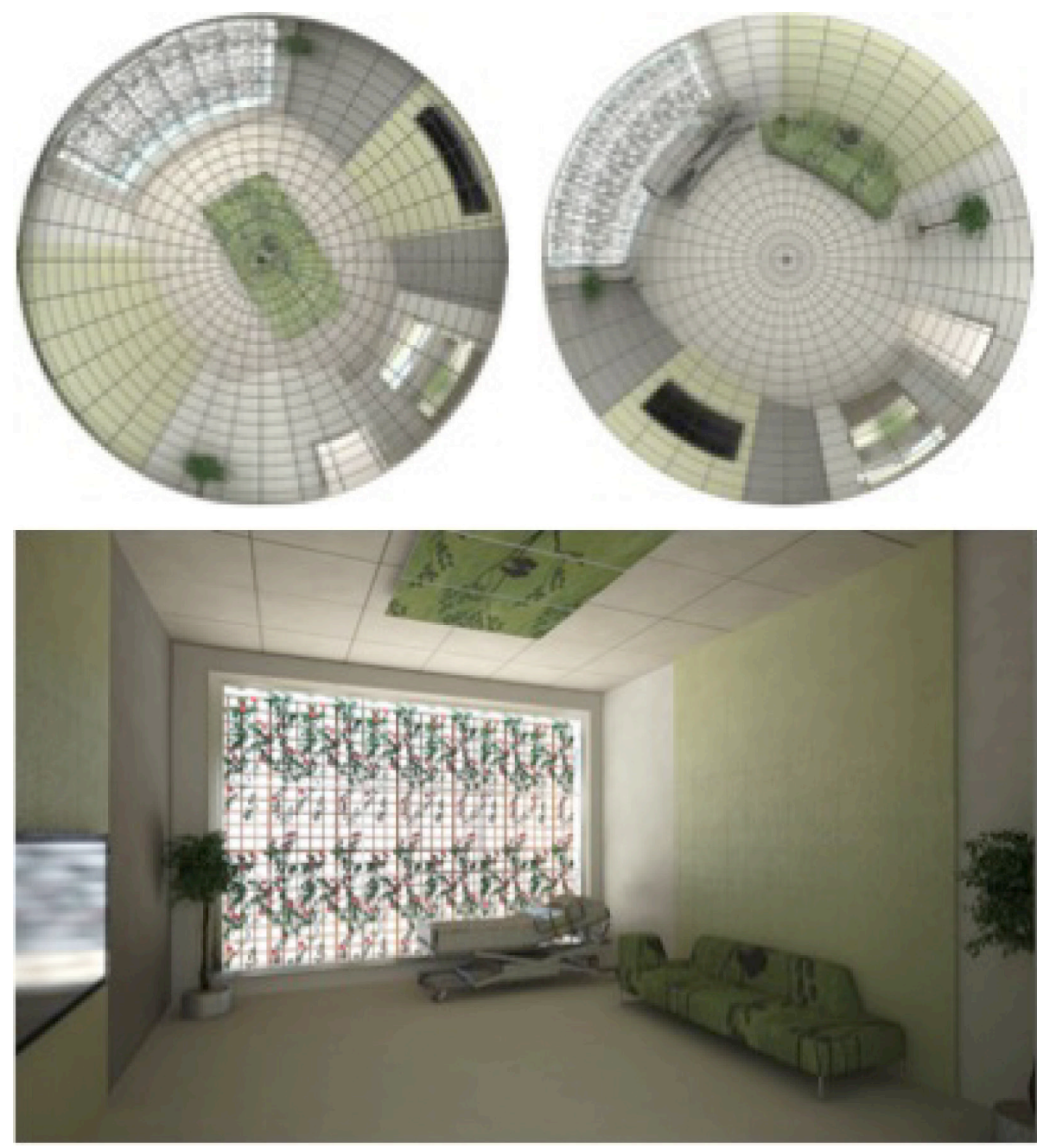

individuals essentially through the choreography of environmental phenomena by means of organizing materials, space, and form. The building scale also addresses the health of communities through both site selection for a given program and volumetric morphology relative to surrounding context and broader density variables. Each scale of research is cross-linked to merge individual and societal human wellbeing benefits into fundamental design principles and application in building design [Fig. 5].

Aspects of the interscalar research outcomes are providing fundamental knowledge areas on human health and wellbeing for built environment design. These outcomes primarily consist of:

1. Science of brain-immune-environmental interactions to inform primary attributes of design strategies;

2. Strategies for improved public health at regional and neighborhood scales resulting from Urban Form Index metrics;

3. Design methods integrating human health and wellbeing attributes in development and configuration of material, space, and form.

Each of these primary outcomes is being translated into fundamental design principles that are introduced in the architecture curriculum through core environmental courses. The design principles emphasize the relationship between environmental phenomena, such as light, air, and sound, to the correlated effects on human experience and benefit. While basic
Figure 4: Fisheye View-Factor Calculation Method for Design Integration of Wellbeing Attributes. (Images: G. Shirazi) 


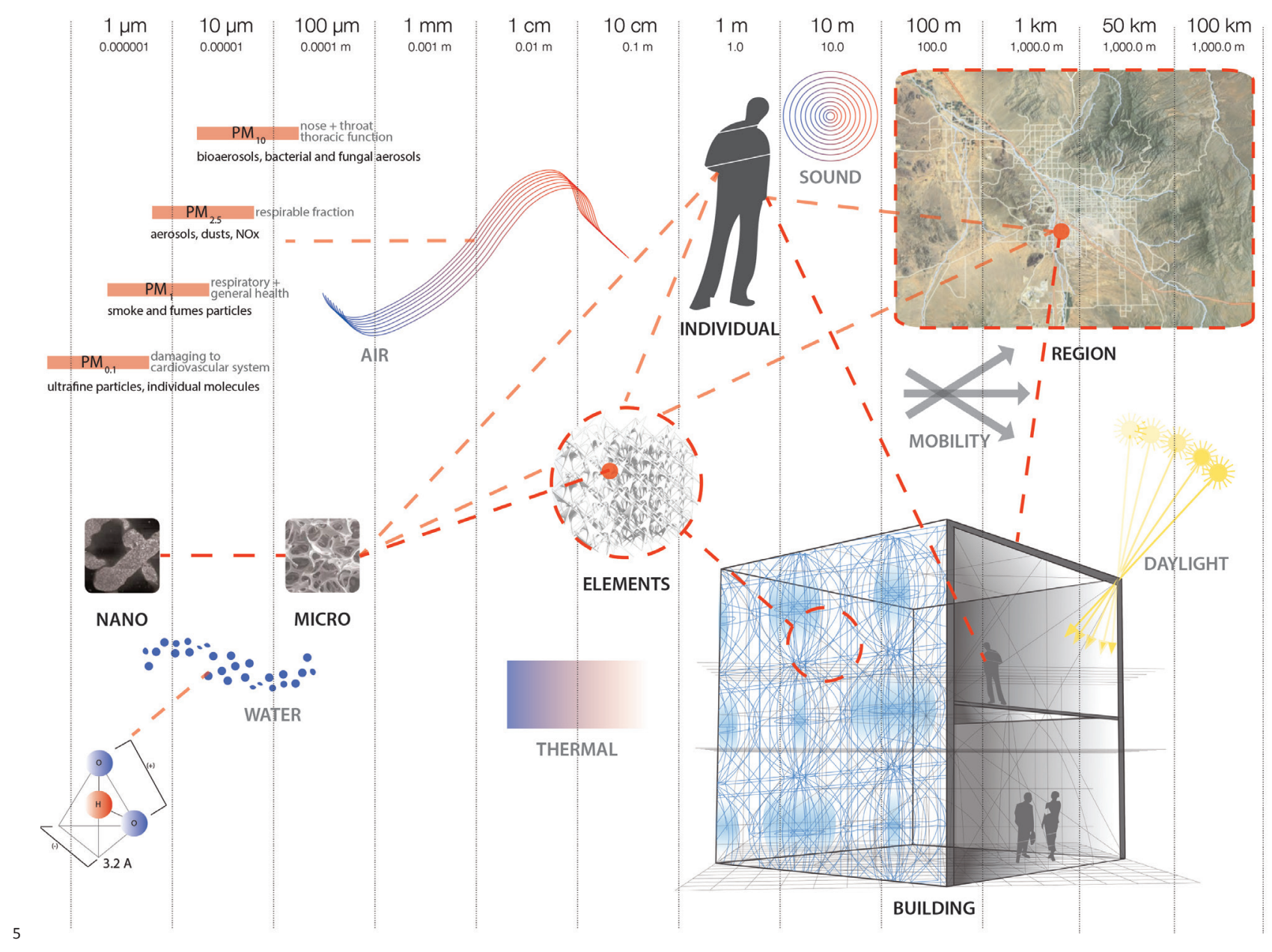

Figure 5: Interscalar Health Attributes for Built Envrionment Design Integration. environmental physics is well established in design research, correlations to human health response begin to inform alternate implications for spatial and formal manifestations.

OIn addition, core fundamentals and design principles for urban form and regional development patterns are established through Urban Form Index metrics to improve public health and quality-of-livability influencing greater societal wellbeing. These community scale programmatic and morphological design principles are introduced in urban design and planning curriculum, as well as in the sustainable built environment courses. These fundamentals also find their way into practice through the outreach activities of our College of Architecture, Planning, and Landscape Architecture, including technical assistance provided to underserved communities facilitated through the college's Drachman Institute.

Our interdisciplinary research team is also an active member of the American Institute of Architect's Design and Health Research Consortium that was inaugurated in January 2015. The partnership established through this consortium is allowing for the opportunity for our human health and built environment research findings and the resultant design principles to be shared with the broader professional design community. The integrated outcomes of the interdisciplinary research directly inform a collective resource influencing living practitioners and policy-makers in future built environment design decisions. 


\section{GOALS AND FUTURE WORK}

The goals for facilitating the ongoing and projected human health and wellbeing interdisciplinary and interscalar design research into curriculum and practice concurrently address:

1. Establishing the methods for cross-correlating human health response to environmental factors with the specifics of building form, space, and material composition as experienced by the individual;

2. Establishing the methods for correlating Urban Form Index public health and livability metrics with community design principles as determinants for collective wellbeing experience;

3. Translating the quantifiable outcomes from these innovative methods to new baseline knowledge informing design principles in the architectural and community/urban design process;

4. Integrating these design principles for health and wellbeing in core fundamental teaching in professional architecture and sustainable built environment curriculum;

5. Applying these core principles in the design and construction of DDBC affordable housing projects for ongoing evaluation and monitoring;

6. Applying these core principles in the planning projects and efforts through the college's Drachman Institute outreach arm; and

7. Introducing these core principles to the broader professional design and policy context through active participation on the AIA Design and Health Research Consortium.

\section{ENDNOTES}

1. J.F. Thayer and E.M. Sternberg, et al. "The Effects of the Physical Work Envrionment on Circadian Variations in Heart Rate Variability and the Morning Rise in Cortisol." European Journal of Cardiovascular Prevention and Rehabilitation, 17(4) Aug 2010, p.431-439.

2. Juhani Pallasmaa. The Eyes of the Skin: Architecture and the Senses. Leinster Gardens, London: Academy Group, Ltd., 1996.

3. http://who.int/about/definition/en/print.html

4. Esther M. Sternberg. Healing Spaces: the Science of Place and Wellbeing. Cambridge, MA: Harvard University Press, 2010.

5. Ibid.

6. Sheldon Cohen, L. Underwood, and B. Gottlieb, Eds. "Social Relationships and Health" in Social Support Measurement and Interventions: A Giude for Health and Social Scientists. New York, NY: Oxford, 2000.

7. John Cacioppo, hand-holding studies from book titled Loneliness.

8. Judith Heerwagen.

9. Ewing and Hamidi were part of a team of research ers comprising the Metropolitan Research Center at the University of Utah when Arthur C. Nelson was its director from 2008-2014.

10. http://gis.cancer.gov/tools/urban-sprawl/

11. For a description, see http://en.wikipedia.org/ wiki/Walk_Score.

12. Ewing and Hamidi, p.19.

13. Term established by Arthur C. Nelson

14. Arthur C. Nelson. Reshaping Metropolitan America. Washington, DC: Island Press, 2013.

15. Arthur C. Nelson. Foundations of Real Estate Development Financing: A Guide to Public-Private Partnerships. Washington, DC: Island Press, 2014.

16. Arthur C. Nelson helped lead a team of researchers at the University of Utah to create Envision Tomorrow Plus as a sophisticated, open-source scenario planning tool that is changing long-range land use planning nationally. See http://www.envisiontomorrow.org/about-envision-tomorrow/. 